DOI: $10.3901 / J M E .2020 .17 .198$

\title{
钢轨波磨对高速列车车轮多边形磨耗 产生与发展的影响“
}

\author{
吴 越 ${ }^{1}$ 韩 健 $^{2}$ 左齐宇 ${ }^{1}$ 金学松 $^{1}$ 肖新标 $^{1}$ 梁树林 $^{1}$
}

(1. 西南交通大学牵引动力国家重点实验室 成都 610031;

2. 西南交通大学机械工程学院 成都 610031)

\begin{abstract}
摘要: 列车车轮多边形磨耗问题广泛存在于我国高速列车上, 会显著增大轮轨之间的相互作用力, 严重影响列车运行安全性 和舒适性, 其产生和发展机理值得探究。车轮多边形磨耗与钢轨波浪形磨损(简称: 波磨)从磨耗特征来看较为相似, 只是发 生磨耗的载体不同, 钢轨波磨是否对车轮多边形磨耗存在根本的影响值得深入研究。以我国某线路上运行的高速动车组列车 为研究对象, 通过建立车轮多边形磨耗仿真预测模型, 结合现场试验数据, 调查钢轨波磨对车轮多边形磨耗产生和发展的影 响。研究表明, 钢轨波磨虽然可以导致轮轨力和蠕滑率等磨耗关键参数沿车轮圆周发生周期性波动, 但是在车辆实际运营条 件下, 钢轨波磨导致车轮多边形磨耗产生这一观点的成立条件极为 “苛刻” , 对车轮和钢轨波磨区段起始点的接触位置、钢 轨波磨的波长以及车轮周长都有严格的要求。在实际中该条件难以满足。因此, 钢轨波磨的存在并不是车轮多边形产生的根 本原因。结果可为高速列车车轮多边形形成机理的相关研究提供参考和指导。
\end{abstract}

关键词: 车轮多边形磨耗; 钢轨波磨; 高速列车; 车辆轨道耦合动力学模型; 机理

中图分类号: U270

\section{Effect of Rail Corrugation on Initiation and Development of Polygonal Wear on High-speed Train Wheels}

\author{
WU Yue $^{1}$ HAN Jian $^{2} \quad$ ZUO Qiyu $^{1} \quad$ JIN Xuesong $^{1} \quad$ XIAO Xinbiao $^{1} \quad$ LIANG Shulin $^{1}$
}

(1. State Key Laboratory of Traction Power, Southwest Jiaotong University, Chengdu 610031;

2. College of Mechanical Engineering, Southwest Jiaotong University, Chengdu 610031)

\begin{abstract}
The polygonal wear of train wheels occurs in rail transport commonly and will increase the wheel-rail interaction force dramatically and have a bad effect on the safety and comfort of the train. The mechanism of the polygonal wear needs to be studied. The polygonal wear looks like the corrugated wear of rail, the former occurs on wheels and the later occurs on rails. So, the relationship between the rail corrugation and polygonal wear deserves further study. The polygonal wear prediction model is developed to study the effect of rail corrugation on initiation and development of polygonal wear. The research shows that the rail corrugation can lead to the periodic fluctuation of key wear parameters such as wheel rail normal force and creep slip rate along the wheel circumference. But, restricted by the randomness of the position of the wheel-rail contact point, the wavelength of the rail corrugation and the circumference of the wheels, it is hard to say that the rail corrugation lead to the polygonal wear on wheels. And the rail corrugation is not the root cause of the polygonal wear on wheels. The results obtained in this investigation may be helpful for the further study on the mechanism of polygonal wear of the wheels.
\end{abstract}

Key words: polygonal wear; rail corrugation; high-speed train; train/track coupling dynamics model; mechanism

\section{0 前言}

列车车轮多边形磨耗是指车轮沿圆周方向上所

* 国家自然科学基金(U1734201, U1934203, 51805450)、四川省科技计划 (2020YJ0254，2020YJ0076)和西南交通大学博士研究生创新基金资助项 目。20191001 收到初稿, 20200401 收到修改稿
发生的非均匀磨耗，其波长分布较广，短至十几厘 米, 长至整个车轮周长。车轮多边形磨耗会引起轮 轨间作用力显著增大，对车辆和轨道部件产生恶劣 的影响, 大大降低系统零部件的使用寿命, 严重时 将会威胁到行车安全。普通列车和高速列车均会出 现车轮多边形磨耗现象 ${ }^{[1-2]}$ 。此问题和钢轨波磨问 
题 ${ }^{[3]}$ 一样, 已经逐渐成为我国乃至世界高速铁路运 输行业中的难题, 引起国内外学者的广泛关注。

$\mathrm{KAPER}^{[4]}$ 在研究短途客运列车噪声问题时, 发 现了车轮多边形磨耗这一现象, 从频域的角度研究 了车轮多边形磨耗所带来的车辆行车噪声问题, 指 出车轮多边形磨耗会使得车内噪声显著提高。 NIELSEN 等 ${ }^{[2]}$ 对车轮多边形磨损问题进行了全面 地研究, 对车轮磨损预测的数值方法进行了文献综 述, 并提出了车轮多边形磨耗的防治措施以及车轮 的锭修准则。MORYS 等 ${ }^{[5-7]}$ 建立了 ICE-1 型高速列 车动力学模型, 模型中将车轮和闸盘视为刚体, 刚 体之间利用三维旋转弹簧-阻尼单元连接, 以表征轮 对的弯曲和扭转特性。轮轨滚动接触采用 Kalker 简 化模型, 并采用长效轮轨磨损模型, 计算在各阶初 始车轮多边形磨耗条件下, 车轮半径随里程的变化 量。通过计算发现, 列车运行时, 车轮多边形磨耗 会导致轮轨法向力波动幅度显著增高, 激发轮对的 弯曲共振模态, 导致轮轨间横向蠕滑力呈周期性变 化, 最终诱发车轮多边形磨耗。MEINKE 等 ${ }^{[8]}$ 将 MORYS 的轮对模型进行了改进, 制动盘和车轮等 刚体之间的连接由弹簧-阻尼单元变为弹性梁单元, 考虑到了轮轴的回转效应。将高速列车车轮垂向振 动问题转化为转子动力学问题, 主要受重力、转动 惯量和回转力矩的影响。运用不平衡计算和长效轮 轨磨损仿真分析车轮多边形磨耗形成和发展过程。

MEYWERK ${ }^{[9]}$ 认为在不平衡动力作用下轮对和钢轨 相互作用下发生的柔性变形导致了车轮多边形磨耗 的形成和发展, 考虑到轮对和钢轨的柔性变形, 建 立了单个弹性轮对在弹性轨道上运行的动力学计算 模型。钢轨和轮对由轮框架导向, 轮对框架常速平 动, 框架和轮对在轮轴末端处通过三个弹簧-阻尼单 元连接。计算结果表明, 轮对的一阶对称垂向弯曲 模态和一阶反对称垂向弯曲模态激振引起车轮的横 向滑移量最大, 对车轮非圆化形成和发展产生较大 地影响, 而列车实际运行时也可能是轮对对称和反 对称模态的相位组合。陈光雄等 ${ }^{[10]}$ 运用 ABAQUS 建立轮轨相互作用的有限元模型, 通过计算发现, 在低于 $150 \mathrm{~Hz}$ 频段内, 车轮和钢轨之间会发生横向 黏-滑弹性振动, 这可能是导致车轮多边形磨耗产生 的重要原因。通过选择恰当的轨枕支撑弹簧刚度或 者控制轮轨摩擦因数可以抑制车轮多边形磨损。JIN 等 ${ }^{[11-12]}$ 对我国广州地铁列车车轮多边形的发生发展 机理进行了调研, 认为轮对一阶弯曲振动是车轮产 生 9 边形磨耗的原因, 轮对的一阶弯曲共振导致轮 对在垂向平面发生弯曲变形, 轮对一阶弯曲振动引
起轮轨接触区车轮相对钢轨横向滑动。轮轨间横向 蠕滑率变化周期与轮轨弯曲共振周期相同, 其会引 起轮轨间横向蠕滑力的周期性变化, 进而导致车轮 发生 9 边形磨耗。吴越等 ${ }^{[13-14]}$ 通过对某 22、23 阶多 边形磨耗车辆开展了系统全面的科学研究试验, 包 括车辆振动测试、转向架模态分析、车轮不圆度测 试、钢轨模态测试和钢轨振动测试, 认为此高速列 车发生 22、23 阶多边形磨耗的根源在于转向架系统 在 $580 \mathrm{~Hz}$ 频率发生模态耦合共振。

通过大量研究, 人们对于高阶车轮多边形磨耗 问题有了一定程度的理解, 并基于不同理论, 提出 了不同的高阶多边形磨耗形成机理。但是这些机理 并不能充分地解释实际中高阶多边形磨耗的特征及 规律, 还值得更加深入地研究。在众多关于车轮多 边形磨耗成因的讨论中, 人们往往将车轮多边形磨 耗地产生和钢轨波磨联系起来, 相关研究人员在正 式或非正式报告中，均提出过钢轨波磨会导致车轮 产生多边形磨耗这一观点。相关部门也花费大量时 间、人力和物力去调查车轮多边形磨耗和钢轨波磨 之间的联系, 然而并没有得到比较确定的结论。另 外, 现有文献中也并无充分证据来表明钢轨波磨和 车轮多边形磨耗之间的关系。实际中, 通过试验来 研究车轮多边形磨耗和钢轨波磨之间的关系几乎不 可能, 因为运营中的高速线路严格地控制波磨发生 和发展, 因此需要借助理论分析的方法进行研究。 故本文以我国某线路上运行的某型高速动车组列车 为研究对象, 通过建立车轮多边形磨耗仿真预测模 型, 结合现场试验数据, 分析在钢轨波磨作用下, 车轮多边形磨耗的产生和发展规律。

\section{1 线路钢轨波磨及车轮多边形情况}

分别对我国某高速铁路线路的钢轨波磨及运行 其上的高速动车组列车的车轮多边形磨耗进行测试, 以获取其波长(或阶次)和幅值。

图 1 给出了此高速铁路线路部分区段的钢轨波 磨测试结果。由图 1 可知, 此线路部分钢轨存在特 征波长为 $151.4 \mathrm{~mm}$ 的钢轨波磨, 在此线路上运行车 辆的车速为 $300 \mathrm{~km} / \mathrm{h}$ 。车辆运行通过钢轨波磨区段 时, 会产生强烈的轮轨异常冲击, 其激励频率可以 由式(1)计算得到

$$
f=\frac{v}{\lambda}=\frac{300}{3.6 \times 0.1514}=550.5
$$

式中, $v$ 为车辆运行速度; $\lambda$ 为钢轨波磨的波长; $f$ 
为激励频率。由式(1)可知, 当列车以 $300 \mathrm{~km} / \mathrm{h}$ 速度 运行通过此波长为 $151.4 \mathrm{~mm}$ 的钢轨波磨区段时, 车 辆会受到 $550 \mathrm{~Hz}$ 频率的载荷作用。

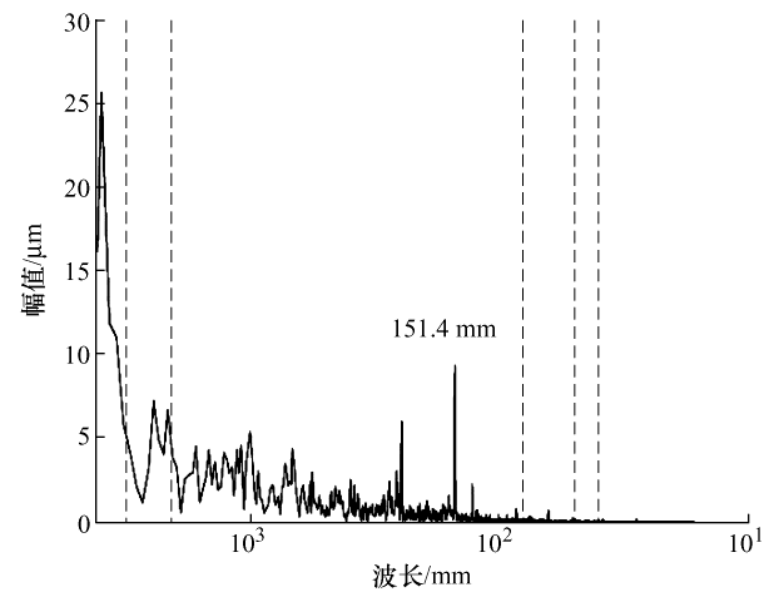

图 1 钢轨波磨测试结果

图 2 给出了运行在此高速线路上的某型动车组 列车全列车轮的多边形磨耗测试结果。

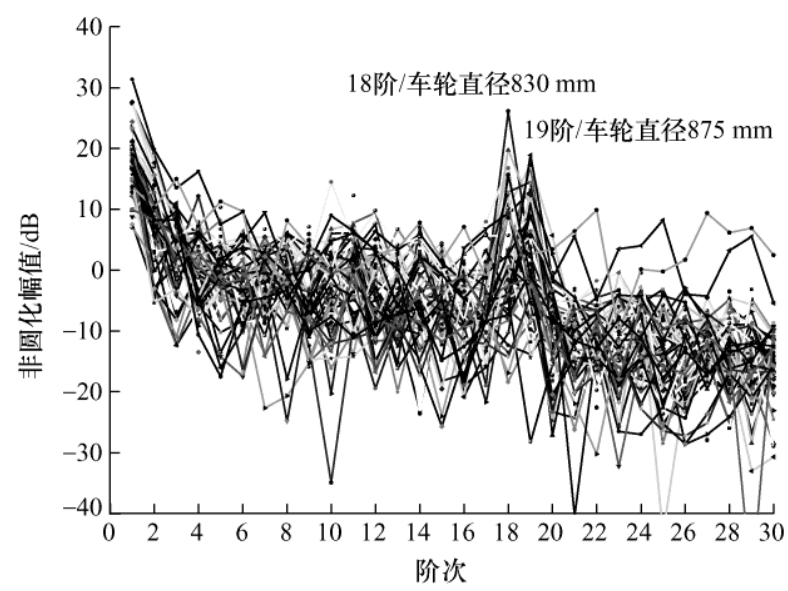

(a) 全列车多边形磨耗测试结果

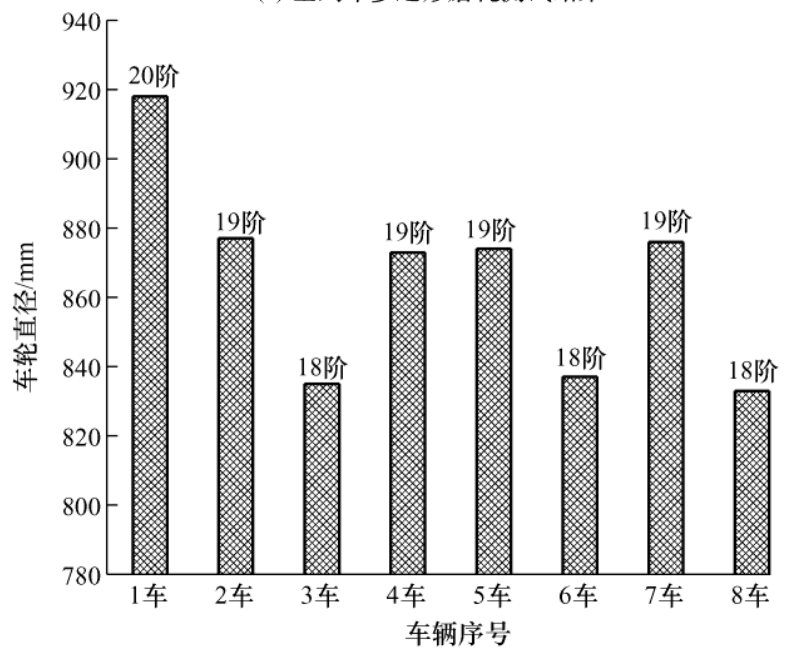

(b) 全列车车轮直径测试结果

图 2 车轮测试结果

由图 2 可知, 此列车主要存在 18 阶和 19 阶车 轮多边形磨耗, 部分车轮存在 20 阶多边形磨耗, 且
出现 19 阶车轮多边形磨耗的车轮直径为 $875 \mathrm{~mm}$, 出现 18 阶车轮多边形磨耗的车轮直径为 $830 \mathrm{~mm}$, 可以说车轮直径和车轮多边形磨耗之间存在一一对 应的关系。通过式(2)计算导致车轮多边形磨耗产生 的激励频率

$$
\left\{\begin{array}{l}
f_{18}=\frac{v}{\pi D} n=\frac{300}{3.6 \times \pi \times 0.830} \times 18=575 \\
f_{19}=\frac{v}{\pi D} n=\frac{300}{3.6 \times \pi \times 0.875} \times 19=576
\end{array}\right.
$$

式中, $f_{18}$ 和 $f_{19}$ 分别为 18 阶多边形磨耗车轮和 19 阶多边形磨耗车轮的激励频率, $v$ 为列车运行速度, $D$ 为车轮直径, $n$ 为车轮阶次。由式(2)计算结果可 知, 虽然不同直径的车轮, 其多边形磨耗阶次不同, 但是它们的激励频率是一致的, 约为 $575 \mathrm{~Hz}$ 。故可 以推测, 车轮多边形磨耗的产生, 和车轮受到固定 频率激励密切相关。

通过对比列车以 $300 \mathrm{~km} / \mathrm{h}$ 运行通过钢轨波磨区 段时所受到激励的频率 $(550.5 \mathrm{~Hz})$ 和导致多边形磨 耗产生的激励频率 $(575 \mathrm{~Hz})$, 发现二者虽然接近但是 仍然相差 $25 \mathrm{~Hz}$, 因此在此条线路上存在的 $151.4 \mathrm{~mm}$ 波长的钢轨波磨是否是运行于此线路上 的列车出现 $18 、 19$ 阶多边形磨耗的根本原因值得深 入研究。另外, 由式(1)反推得出, 钢轨若存在特征 波长为 $144.9 \mathrm{~mm}$ 的钢轨波磨, 此时激励频率为 $575 \mathrm{~Hz}$ ，和多边形磨耗的激励频率完全吻合，那么 是否会导致多边形磨耗产生(虽然此条线路上无 $144.9 \mathrm{~mm}$ 的钢轨波磨)。在实际线路条件下，钢轨 波磨的波长是不可能进行人为控制的, 因此针对上 述问题，需要通过仿真计算分析开展研究。

\section{2 计算模型}

建立车轮多边形磨耗预测模型，以钢轨表面谐 波不平顺模拟钢轨波磨，计算分析在钢轨波磨激励 条件下，车轮多边形磨耗产生和发展规律。

\section{1 车辆-轨道刚柔耦合动力学模型}

图 3 给出了车辆-轨道刚柔耦合动力学模型 ${ }^{[15]}$ 示意图。对于车辆系统来说, 高速列车车辆可以简 化为由 1 个刚性车体、2 个刚性构架、 4 个柔性轮对 组成的刚柔耦合动力学系统。本文中, 仍然将车体 和构架考虑为刚体, 每个刚体考虑了 5 个自由度, 分别为垂向、横向、点头、摇头和侧滚, 共计 15 个自由度。轮对考虑为柔性体, 用有限元实体单元 建模, 并利用模态叠加法求解 ${ }^{[16]}$ 。一系、二系悬挂 系统采用三维黏弹性弹簧-阻尼单元模拟。对于轨道 系统, 采用整体混凝土道床轨道系统, 由钢轨、扣 
件系统、轨道板及路基组成。其中左右钢轨被视为 连续弹性离散点支承基础上的 Timoshenko 梁, 轨道 板轨道考虑成柔性体, 用有限元实体单元建模, 并 利用模态叠加法进行求解 ${ }^{[17]}$ 。扣件系统用三维黏弹 性弹簧-阻尼单元拟, 路基支撑层简化为均匀分布的 弹簧-阻尼单元连接。

本文通过建立轮对有限元模型的方式, 将轮对 考虑为柔性体 ${ }^{[16]}$ 。根据三维实体有限元理论, 轮对 的振动微分方程为

$$
\boldsymbol{M}_{i}\left\{\ddot{\boldsymbol{u}}_{i}(t)\right\}+K_{i}\left\{\boldsymbol{u}_{i}(t)\right\}=\left\{\boldsymbol{Q}_{i}(t)\right\} \quad i=1,2,3,4
$$

式中, $\ddot{\boldsymbol{u}}_{i}(t)$ 和 $\boldsymbol{u}_{i}(t)$ 分别为 $t$ 时刻第 $i$ 位轮对上节点 的加速度矢量和速度矢量, $\boldsymbol{M}_{i}$ 和 $\boldsymbol{K}_{i}$ 分别为第 $i$ 位 轮对的质量矩阵和刚度矩阵, $\boldsymbol{Q}_{i}(t)$ 为 $t$ 时刻第 $i$ 为 轮对所受外力矢量, 对于轮对来说主要受到一系悬 挂力和轮轨相互作用力。

利用模态叠加法求解方程式(3), 令

$$
\begin{gathered}
\boldsymbol{u}_{i}(t)=\boldsymbol{\Phi}_{i} \boldsymbol{q}_{i k}(t) \\
\boldsymbol{\Phi}_{i}=\left(\{\phi\}_{i 1},\{\phi\}_{i 2},\{\phi\}_{i 3}, \ldots,\{\phi\}_{i N}\right)
\end{gathered}
$$

$$
\boldsymbol{q}_{i k}(t)=\left(q_{i 1}(t), q_{i 2}(t), q_{i 3}(t), \cdots, q_{i N}(t)\right)
$$

式中, $\boldsymbol{\Phi}_{i}$ 为第 $i$ 位轮对的正则振型矩阵, $\{\boldsymbol{\phi}\}_{i k}$ 为第 $i$ 位轮对的第 $k$ 阶振型矢量, $\boldsymbol{q}_{i k}(t)$ 为 $t$ 时刻第 $i$ 位 轮对的第 $k$ 阶正则坐标, $N_{i}$ 为考虑的模态阶数。

将式(4)代入式(3), 有

$$
\boldsymbol{M}_{i} \boldsymbol{\Phi}_{i} \ddot{\boldsymbol{q}}_{i k}(t)+\boldsymbol{K}_{i} \boldsymbol{\Phi}_{i} \boldsymbol{q}_{i k}(t)=\left\{Q_{i}(t)\right\} \quad i=1,2,3,4
$$

对方程式(7)两边同时左乘 $\boldsymbol{\Phi}_{i}^{\mathrm{T}}$, 有

$$
\ddot{\boldsymbol{q}}_{i k}(t)+\Omega \boldsymbol{q}_{i k}(t)=\boldsymbol{\Phi}_{i}^{\mathrm{T}}\left\{\boldsymbol{Q}_{i}(t)\right\} \quad i=1,2,3,4
$$

式中, $\Omega=\left(\omega_{1}^{2}, \omega_{2}^{2}, \omega_{3}^{2}, \cdots, \omega_{N}^{2}\right)$ 为各阶模态所对应的圆 频率。最终, 方程可以变为

$$
\ddot{\boldsymbol{q}}_{i k}(t)+\omega_{k}^{2} \boldsymbol{q}_{i k}(t)=\left\{\boldsymbol{\phi}_{i k}\right\}^{\mathrm{T}}\left\{\boldsymbol{Q}_{i}(t)\right\} \quad i=1,2,3,4
$$

运用显示积分算法可以求解上述振动微分方程, 得到模态坐标下的节点位移和节点加速度, 进而由 式(4)求得惯性坐标系下的节点位移、速度和加速度。

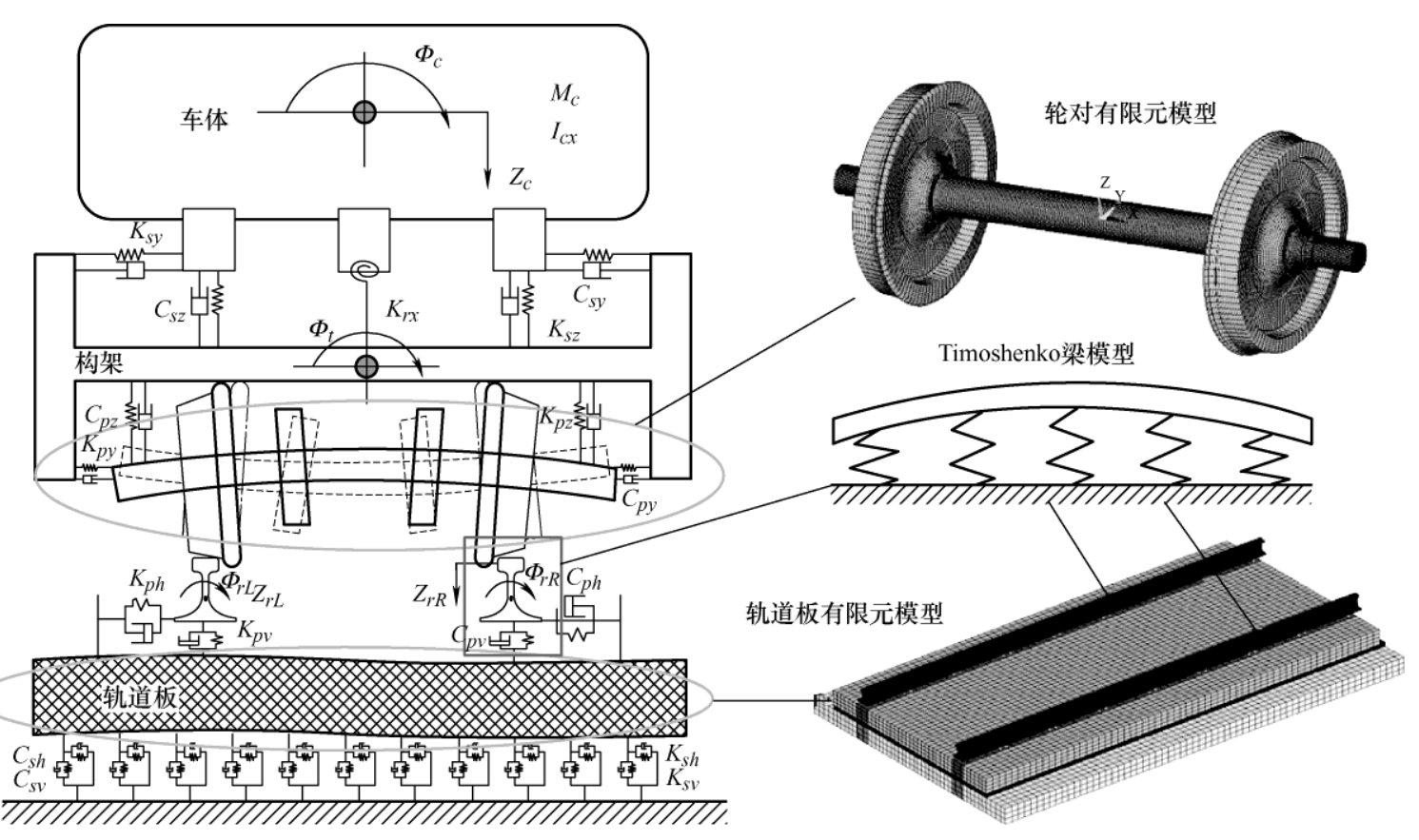

图 3 刚柔耦合动力学模型

本文主要采用实时在线获取轮轨接触点方法 ${ }^{[18]}$ 来确定柔性轮对接触关系。对于柔性轮对的运动主 要包含刚性运动和柔性变形, 刚性运动主要是指轮 对的平移运动、侧滚运动和摇头运动。柔性变形在 本文主要体现在轮轴的弯曲变形上。当轮对进行刚 性运动和轮轴弯曲变形后, 左右车轮分别采用 2 个 虚拟的刚性轮表征, 通过求解虚拟轮对中心在轨道 坐标系的位移矢量, 再利用迹线法获得虚拟轮对与 钢轨的接触几何关系, 这样将柔性轮对接触问题转
化为刚性轮对接触问题进行求解。在确定了两个虚 拟刚性轮对和钢轨空间几何接触关系之后, 利用迹 线法求得轮轨接触点位置。利用赫兹非线性接触理 论计算轮轨法向力, 利用 Kalker 线性接触理论和沈 氏理论计算轮对切向力。这些理论不在本文赘述, 详见文献[15]。将所求得轮轨力和蠕滑率等参数输 入磨耗模型, 计算车轮踏面的磨耗量。

\subsection{Archard 磨耗模型}

采用 Archard 模型求得整个接触斑内的磨耗量, 
磨耗模型的定义为

$$
V_{\text {wear }}=k_{w} \frac{N d}{H}
$$

式中, $V_{\text {wear }}$ 为接触斑内的磨耗材料体积; $N$ 为法向 接触力; $d$ 为滑动距离 $H$ 为两接触物体中较软物体 的材料硬度; $k_{w}$ 为量纲一的磨耗系数。

如图 4 所示, 把接触斑分割成大小相等的单元, 每个单元的长度为 $\Delta x$ 和 $\Delta y$, 单元受到法向力 $p_{z}$ 作用, 单元纵向和横向滑动速度分别为 $S_{x}$ 和 $S_{y}, a$ 和 $b$ 分 别为接触斑长短半轴。

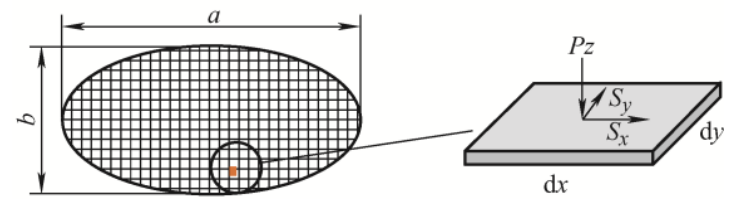

图 4 接触斑内受力计算示意图

对接触斑内的任一单元, 其磨耗深度

$$
\Delta z=k_{w} \frac{p_{z} \Delta d}{H}
$$

式中, 任一单元所受法向力可以表示为

$$
p_{z}=p_{z}(x, y)=\frac{3 N}{2 \pi a b} \sqrt{1-\left(\frac{x}{a}\right)^{2}-\left(\frac{y}{b}\right)^{2}}
$$

滑动距离可以表示为

$$
\Delta d=|s| \Delta t=\sqrt{S_{x}^{2}+S_{y}^{2}} \frac{\Delta x}{v_{c}}
$$

式中, $v_{c}$ 为单元通过接触斑的速度。将式(12)和式(13) 代入式(11), 可得接触斑内任意单元的磨耗量为

$$
\Delta z(x, y)=\frac{3 N k_{w}}{2 \pi a b H} \sqrt{1-\left(\frac{x}{a}\right)^{2}-\left(\frac{y}{b}\right)^{2}} \sqrt{S_{x}^{2}+S_{y}^{2}} \frac{\Delta x}{v_{c}}
$$

式中, $(x, y)$ 表示接触斑内单元的位置, 忽略因弹性 变形产生的速度分量, 并假定滚动接触斑局部为轻 微滑动, 式(14)可简化为

$$
\begin{gathered}
\Delta z(x, y)= \\
\frac{3 N k_{w} \Delta x}{2 \pi a b H} \sqrt{1-\left(\frac{x}{a}\right)^{2}-\left(\frac{y}{b}\right)^{2}} \sqrt{(\xi-\phi x)^{2}+(\eta+\phi y)^{2}}
\end{gathered}
$$

式中, $\xi 、 \eta$ 和 $\phi$ 分别为纵向蠕滑率、横向蠕滑率和 自旋蠕滑率, 这些参数以及正压力、接触斑长短半 轴等均通过动力学程序计算得到。从式(15)中可以 看出, 接触斑内磨耗量大小和正压力及蠕滑率有密 切关系, 当这些参量在车轮圆周上的分布存在周期 性波动的时候, 会导致磨耗量发生周期性波动, 使 得车轮沿圆周方向出现 “多边形” 特征。

磨耗系数的值与轮轨间的法向接触压应力和滑 动速度有关。在模拟磨耗中, 磨耗系数是十分重要 的, 这是因为它提供了在磨耗表达式中引入非线性
特性的可能性。磨耗系数随接触压应力和滑动速度 的变化情况如图 5 所示。

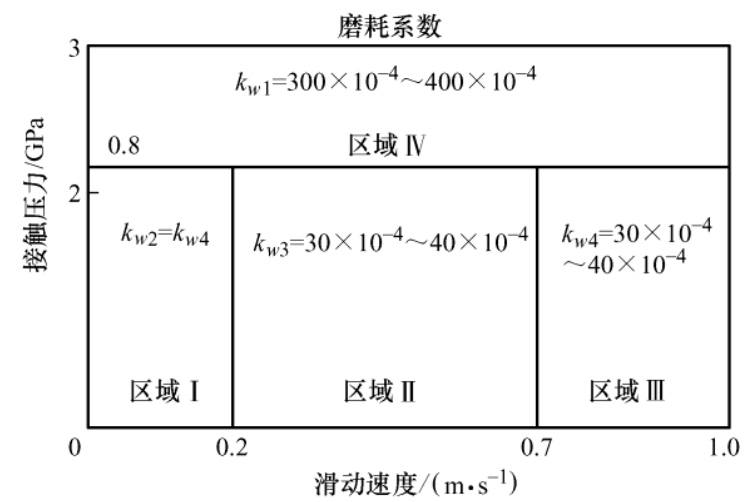

图 5 磨耗系数随接触压应力和滑动速度的变化

在计算得到每一时刻接触斑内的磨耗量后, 为 了把每一时刻计算的接触斑内的磨耗量叠加到车轮 圆周上, 需要将车轮整个圆周的型面离散展开, 如 图 6 所示, 横坐标为展开后的车轮圆周方向坐标, 坚坐标为踏面方向。固定不动的是型面网格, 而在 型面网格间不断运动的就是接触斑。也就是说, 车 轮同时发生踏面方向和周向两个方向的磨耗，本文 仅研究周向磨耗。由于每一个积分步里面求得的接 触斑内的磨耗量也是离散的网格, 这两个网格不会 重合, 这里采用了插值的方法, 将接触斑内的磨耗 映射到车轮型面离散网格。以名义滚动圆位置的磨 耗来表征周向的车轮多边形磨耗。

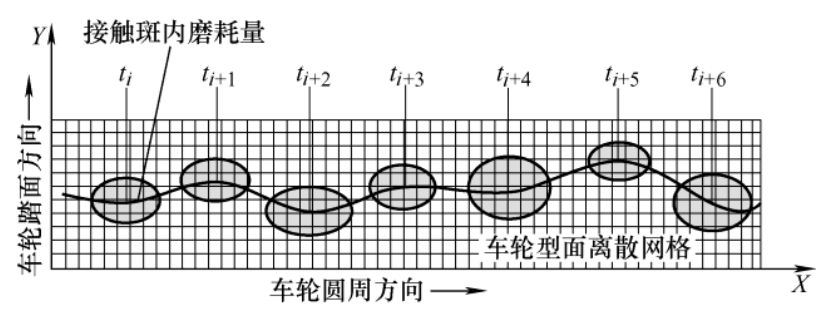

图 6 接触斑磨耗量叠加到车轮圆周示意图

本文对于多边形磨耗的计算, 没有考虑因车轮 踏面发生磨耗而发生变化, 即没有进行车轮型面更 新, 计算结果仅用于定性分析车轮多边形磨耗的发 展规律。

\section{3 钢轨波磨激励下的车轮多边形磨耗 特征研究}

由上文分析可知, 轮轨力和蠕滑率沿车轮圆周 方向发生周期性波动会导致磨耗量的周期性波动, 从而产生多边形磨耗。而轮轨间不平顺作为主要激 励源, 是影响轮轨力和蠕滑率的关键因素。本文设 
置如图 7 所示的计算工况, 研究车辆运行于波磨的 钢轨上时, 车轮是否会发生多边形磨耗。

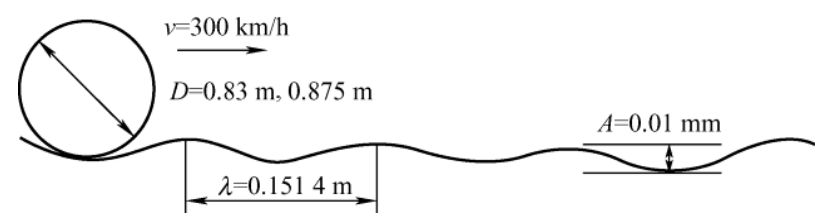

图 7 计算工况示意图

列车运行速度 $v$ 为 $300 \mathrm{~km} / \mathrm{h}$, 钢轨波磨的波长 $\lambda$ 为 $151.4 \mathrm{~mm}$, 波深 $A$ 为 $0.01 \mathrm{~mm}$, 列车车轮直径 $D$ 分别为 $830 \mathrm{~mm}$ 和 $875 \mathrm{~mm}$, 初始的车轮为理想圆。

\section{1 钢轨波磨对轮对动力学特性的影响}

车辆运行时, 因钢轨存在波磨, 会导致其轮对 动力学特性发生变化。图 8 给出了列车以图 7 所示 工况运行时, 轮对的动力学特性计算结果。其中, 图 $8 \mathrm{a}$ 为轮轨法向力计算结果, 图 $8 \mathrm{~b}$ 为蠕滑率计算 结果。图 8 中的横坐标均为运行距离。

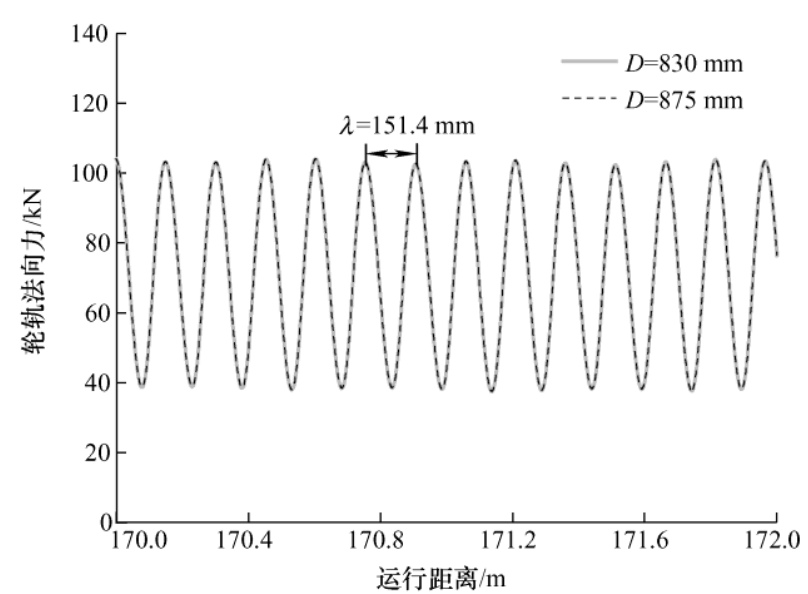

(a) 轮轨法向力

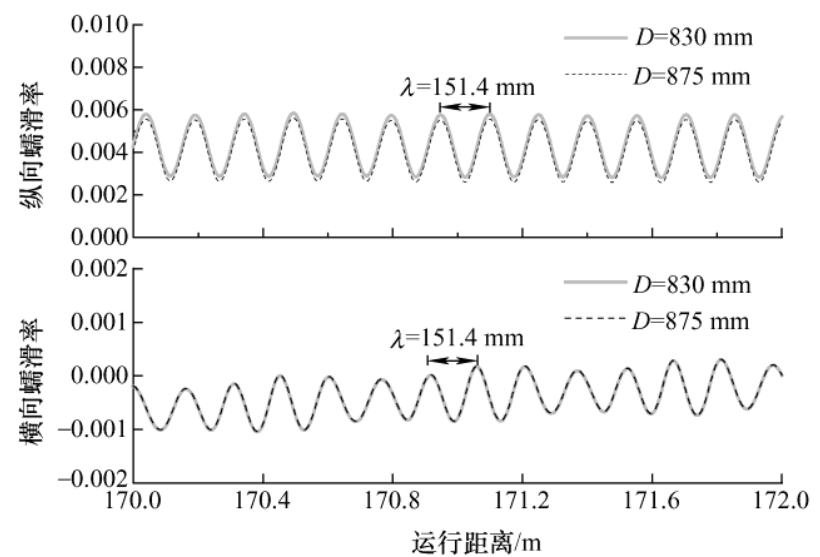

(b) 蠕滑率

图 8 轮对动力学特性计算结果

由图 8 可知, 无论是直径为 $830 \mathrm{~mm}$ 的轮对还 是直径为 $875 \mathrm{~mm}$ 的轮对, 车辆受到钢轨波磨的激 励是一致的。这里需要注意, 由于采用的钢轨波磨
激励比较大, 因此轮轨相互作用主要由钢轨波磨的 特征来决定的。当车辆运行于钢轨波磨区段时, 轮 轨法向力和蠕滑率均呈现出随运行里程的波动特性, 且波长均为 $151.4 \mathrm{~mm}$, 这个钢轨波磨的特征波长保 持一致。因此, 可以认为在钢轨波磨激励下, 轮轨 法向力和蠕滑率等影响磨耗量大小的这些关键参数, 在车轮圆周上的分布也具有周期性波动的特征, 因 此有可能导致磨耗量沿着车轮圆周也呈周期性波动, 关键是这种周期性磨损的波深随着运营里程的增加 会迅速增加。

\section{2 车轮多边形磨耗特征}

以第 3.1 节中计算得到的轮轨法向力和蠕滑率 为输入, 基于 Archard 磨耗模型, 计算车轮的多边 形磨耗。图 9 给出了车轮在波磨钢轨上运行 50 圈以 后, 磨耗量沿圆周的分布特性, 计算结果以极坐标 的形式来表示。其中, 图 9a 为直径 $830 \mathrm{~mm}$ 车轮的 多边形磨耗计算结果, 图 $9 \mathrm{~b}$ 为直径 $875 \mathrm{~mm}$ 车轮的 多边形磨耗计算结果。

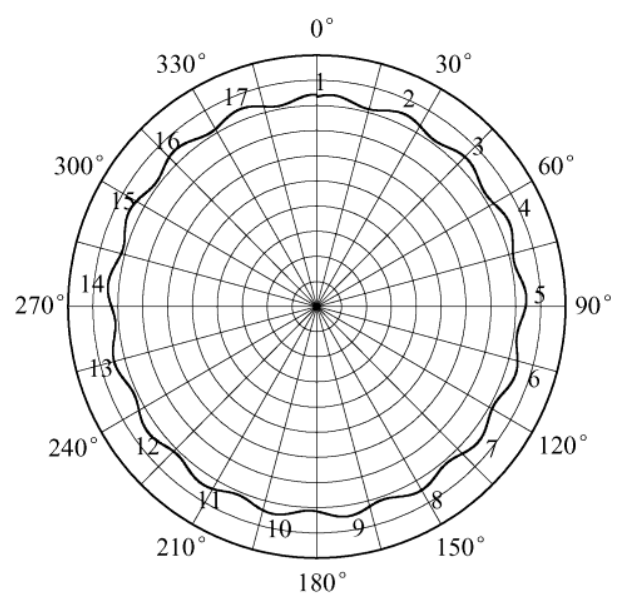

(a) $D=830 \mathrm{~mm}$

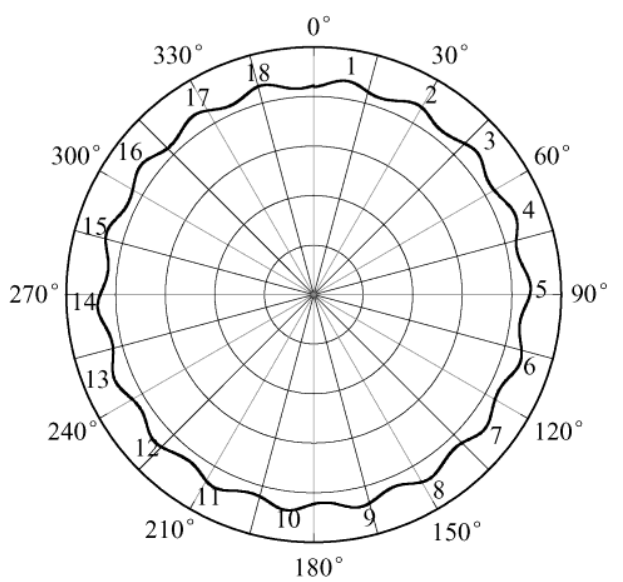

(b) $D=875 \mathrm{~mm}$

图 9 车轮多边形磨耗计算结果

由多边形磨耗计算结果可知, 直径为 $830 \mathrm{~mm}$ 的车轮发生的是轻微的 17 阶多边形磨耗, 直径为 
$875 \mathrm{~mm}$ 的车轮发生的是轻微的 18 阶多边形磨耗, 这和运行于此线路上车辆的多边形磨耗测试结果略 有差异(主要指阶数上的差异)。由上文所示的测试 结果可知, 直径为 $830 \mathrm{~mm}$ 的车轮主要存在 18 阶多 边形磨耗, 而直径为 $875 \mathrm{~mm}$ 的车轮主要存在 19 阶 多边形磨耗。因此, 说明此线路上的钢轨波磨 (151.4 mm 波长)并不是导致车轮发生多边形磨耗的 根本原因。

虽然和实测结果有所差异, 但是可以看出当 车辆运行于存在波磨的钢轨区段时, 轮轨法向力 和蠕滑率沿圆周方向呈周期性分布, 车轮仍会出 现一定程度的多边形磨耗。因此, 还需要进一步 研究在钢轨波磨激励下, 车轮多边形磨耗的发展 规律。

\section{4 钢轨波磨激励下的车轮多边形磨耗 发展关键条件分析}

列车在波磨钢轨上运行时, 会使得车轮产生一 定程度的多边形磨耗。但是多边形磨耗的波深是否 能快速发展起来，仍需要注意到这三点问题。

（1）实际中，一条线路上往往存在着多段区间 的钢轨波磨, 并且它们的波长也不尽相同。除此之 外, 即便是同一段钢轨波磨, 其波长也不为固定值, 沿钢轨方向其波长会存在一定程度的差异。

(2) 按照文献[13]中所述, 轮轨激励的波长满足 恰好整分车轮周长时, 多边形磨耗发展最迅速, 这 里就对波磨波长和车轮直径之间的匹配关系有要求。 事实上, 钢轨波磨的波长和车轮周长不一定满足整 除关系。

（3）很少有波磨存在于整条线路，一般情况下， 钢轨波磨仅存在于线路的某一段。因此每次列车 运行通过时, 车轮和波磨区段起始位置的接触点 分布也是随机的。本节主要针对这三个问题展开 详细讨论。

\section{1 波长差异的影响}

为研究当线路上钢轨波磨的波长不再为一恒 定值时, 对车轮多边形磨耗发展的影响, 现假定 此线路上连续存在 6 种波长接近但仍略有差异的 钢轨波磨, 其波长分别为 $153.4 \mathrm{~mm} 、 151.4 \mathrm{~mm}$ 、 $149.9 \mathrm{~m} 、 148.2 \mathrm{~mm} 、 146.5 \mathrm{~mm}$ 和 $144.9 \mathrm{~mm}$, 每段 长度相同。计算直径为 $830 \mathrm{~mm}$ 的车轮运行通过时, 其多边形磨耗发展情况, 并和相同里程下, 线路 上为单一波长波磨时的多边形磨耗发展情况进行 对比, 单一波长为 $144.9 \mathrm{~mm}$ 。计算工况示意图如
图 10 所示。

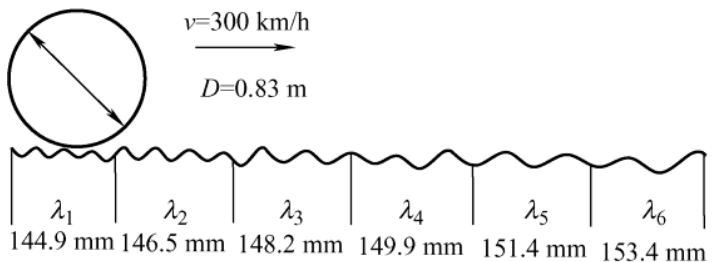

(a) 混合波长

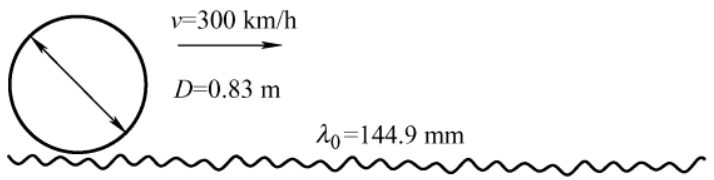

(b) 单一波长

图 10 “混合波长”和 “单一波长”计算示意图

图 11 给出了两种工况下, 车轮滚动相同里程后 的多边形磨耗情况。由图可知, 当车辆运行于 “混 合波长” 的钢轨波磨区段时，其车轮多边形磨耗水 平相较于车辆运行于 “单一波长” 的钢轨波磨区段 时要轻微得多, 发展十分缓慢。

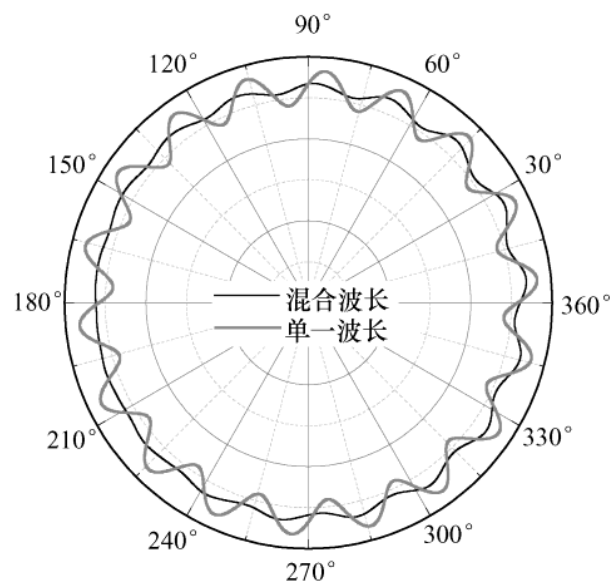

图 11 “混合波长” 和 “单一波长” 计算结果对比

特别注意的是, 这里所设置的 “混合波长”，仅 考虑了 6 种波长, 此 6 种波长各自的差异均不超过 $10 \mathrm{~mm}$, 并且每种波长的波磨区段长度相等, 而实 际中无论是波长还是每种波磨区段的长度以及其分 布均是随机的，其波长的偏差范围可能更大，这些 情况导致车轮多边形磨耗更加不容易完全重叠累积 起来, 这样的话, 多边形磨耗的波深就不可能很大 或发展很快。

\section{2 钢轨波磨波长和车轮周长整分关系的影响}

在文献[13]中提到车轮周长和激励波长之间满 足整分关系时，多边形磨耗发展最迅速，如图 12 所示。此结论得到测试现场大多数试验数据的论证, 具有很高的可信度。列车运行通过钢轨波磨区段时, 
所受到激励的波长即为钢轨波磨的波长。因此, 需 要保证钢轨波磨的波长和车轮周长之间满足整分关 系，车轮多边形磨耗才会发展起来。

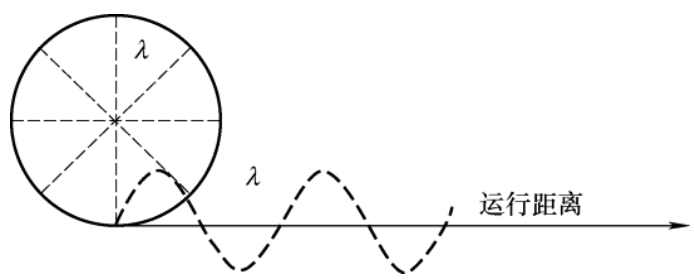

图 12 频率固定激励示意图

为研究钢轨波磨的波长和车轮周长之间的整分 关系对车轮多边形磨耗发展的影响, 现计算直径为 $830 \mathrm{~mm}$ 的车轮运行通过波长为 $144.9 \mathrm{~mm}$ 的钢轨波 磨区段, 其多边形磨耗情况, 并和上文计算所得的 同样直径的车轮运行通过波长为 $151.4 \mathrm{~mm}$ 的钢轨 波磨区段, 其多边形磨耗情况进行对比。图 13 给出 了运行同样里程后, 两种工况下的磨耗量分布特性 对比结果。

由图 13 可知, 对于直径为 $830 \mathrm{~mm}$ 车轮的列 车, 运行通过 $144.9 \mathrm{~mm}$ 特征波长的钢轨波磨区段 时, 出现了显著的 18 阶多边形磨耗。而运行通过 $151.4 \mathrm{~mm}$ 特征波长的钢轨波磨区段时, 多边形磨 耗并不显著。

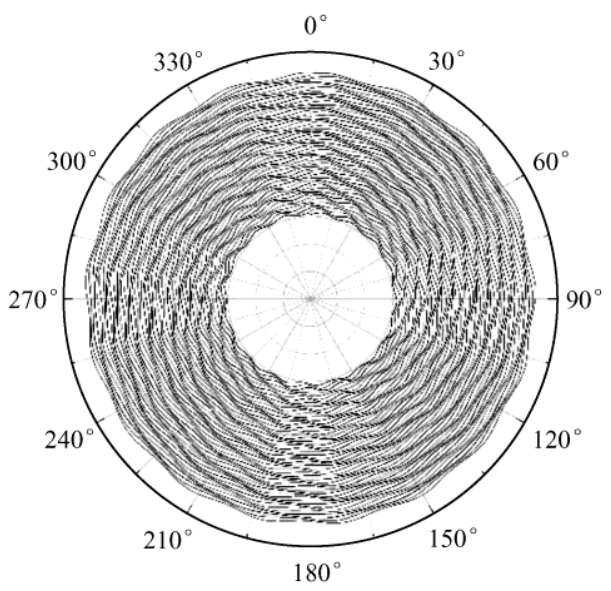

(a) $\lambda=151.4 \mathrm{~mm}$

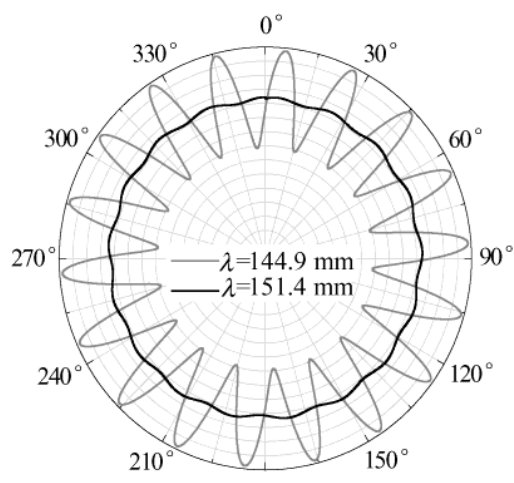

图 13 车轮多边形磨耗对比

利用式(16)也可算得, 当波长为 $144.9 \mathrm{~mm}$ 时, 恰好能 18 整分车轮周长, 多边形磨耗发展迅速。而 波长为 $151.4 \mathrm{~mm}$ 时, 则无明显的整分关系, 多边形 磨耗发展十分缓慢。

$$
\begin{aligned}
& n_{144.9 \mathrm{~mm}}=\frac{\pi D}{\lambda}=\frac{\pi \times 830}{144.9}=18.00 \\
& n_{151.4 \mathrm{~mm}}=\frac{\pi D}{\lambda}=\frac{\pi \times 830}{151.4}=17.22
\end{aligned}
$$

为探明列车通过不同波长的钢轨波磨区段时出 现多边形磨耗程度差异的原因, 现对多边形磨耗发 展过程进行了分析计算, 两种工况车轮滚过 50 圈, 计算结果如图 14 所示, 两种工况所示的多边形磨耗 差异甚大。这个结果说明, 轮轨激励波长严格整除 车轮滚动圆周长, 多边形波深深度才会快速发展。

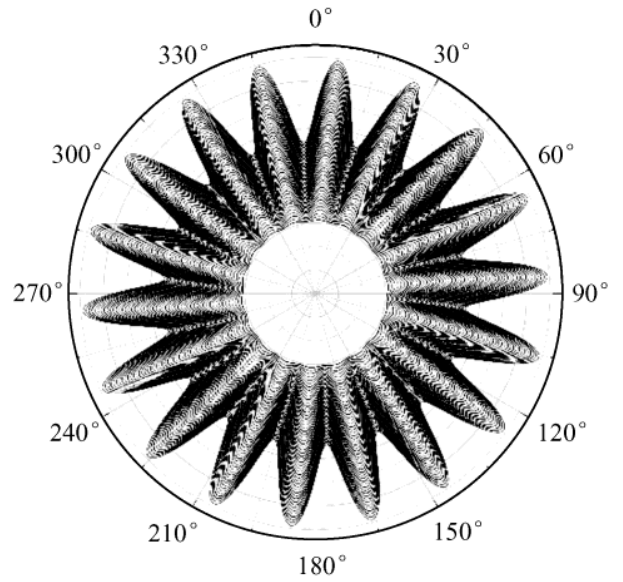

(b) $\lambda=144.9 \mathrm{~mm}$

图 14 车轮多边形磨耗发展情况

\section{3 车轮和波磨起始位置接触点分布随机性的影响}

在第 4.1 节和 4.2 节的计算中, 均假定车辆一直 在钢轨波磨上运行, 进行多边形磨耗叠加和累积。 实际中的钢轨波磨仅存在于线路的某一段或者某几 段区间上, 不同区段的波磨波长可能不同。车辆每 次(或每天重复)通过钢轨波磨区段时, 车轮和钢轨 波磨区段起始位置的接触点在车轮圆周上的分布是
随机的, 即车轮上已有的不均匀磨损和钢轨波磨的 峰谷发生接触的位置具有一定的随机性, 前后相邻 不均磨损不相似, 每一次不均磨耗叠加时可能具有 峰谷相互抵消效应。因此, 每一次多边形磨耗的累 积过程也随之发生了变化。

为研究车轮和波磨起始位置接触点分布随机性 的影响, 现在设置如图 15 所示的计算工况。 


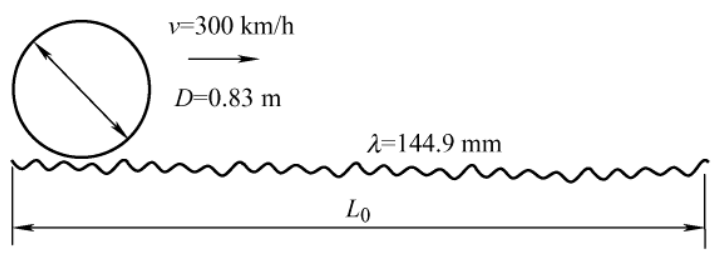

(a) 计算工况a
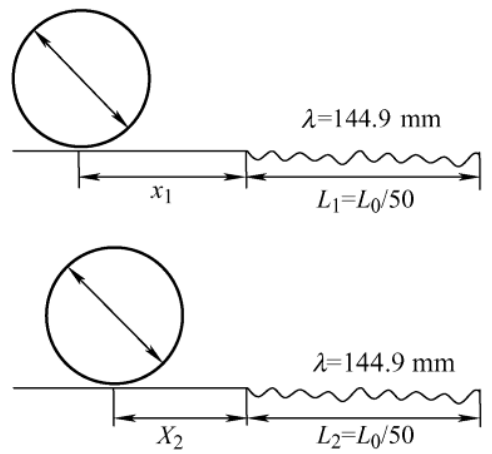

$x_{1}, x_{2}, \cdots, x_{50}$

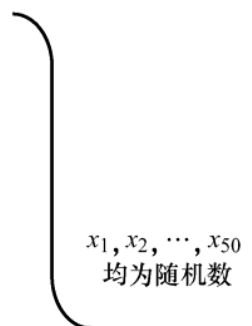

$L_{1}+L_{2}+\cdots+L_{50}=L_{0}$

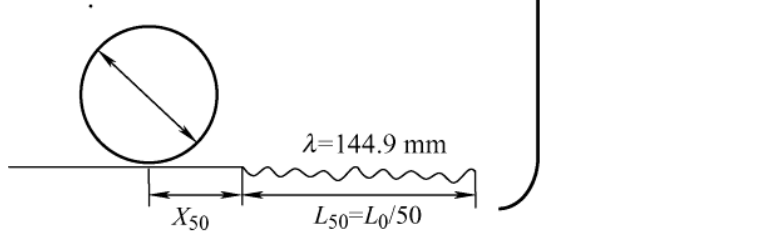

图 15 计算示意图

计算工况 $\mathrm{a}$ 为车轮运行通过一段长度为 $L_{0}$ 的存 在波磨的钢轨, 计算其多边形磨耗情况; 计算工况 $\mathrm{b}$ 为车轮连续运行通过一段长度为 $x_{i}$ 的表面状态正 常的钢轨和长度为 $L_{0} / 50$ 的存在波磨的钢轨, 仅当 车轮进入波磨区段时进行多边形磨耗计算。通过设 置 $x_{i}$ 为随机数的方式, 来表征车轮和波磨起始位置

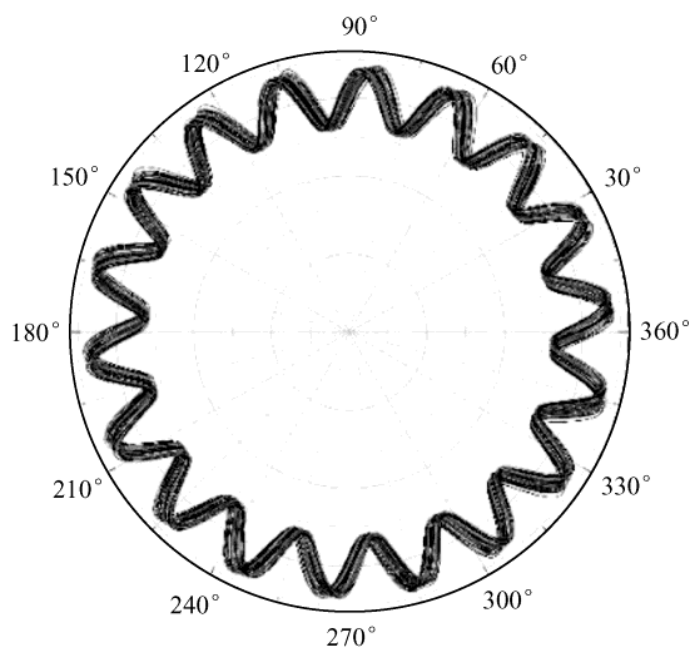

(a) 工况a
接触点分布的随机性, 这样进行 50 次计算, 并将每 次计算得到多边形磨耗结果进行累加, 保证两种工 况下车辆运行相同的里程。两种工况下钢轨波磨的 波长均为 $144.9 \mathrm{~mm}$, 车轮直径均为 $830 \mathrm{~mm}$ 。

对比工况 $\mathrm{a}$ 和工况 $\mathrm{b}$ 的车轮多边形磨耗情况, 结果如图 16 所示。运行相同的距离, 若是考虑到车 轮和波磨起始位置接触点分布的随机性, 则车轮多 边形磨耗不易发展起来。

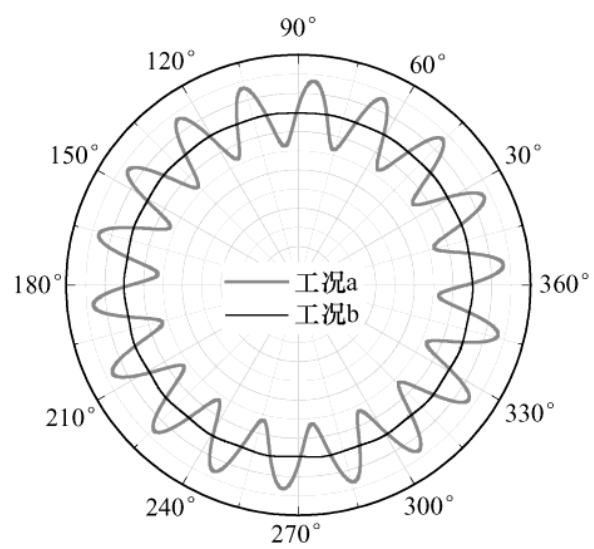

图 16 结算结果对比

为调查导致两种工况下磨耗量出现差异的原因, 现对每次车轮多边形磨耗沿车轮圆周的相位分布展 开讨论, 结果如图 17 所示。工况 $\mathrm{b}$ 条件下每次进行 叠加的磨耗量相位都是随机的, 这样会出现磨耗波 动量抵消的现象, 使得多边形磨耗不容易累积叠加 起来。而工况 a 条件下磨耗量的相位关系比较一致, 这样利于磨耗量沿着车轮圆周方向的快速叠加, 即 多边形磨耗深度快速发展。

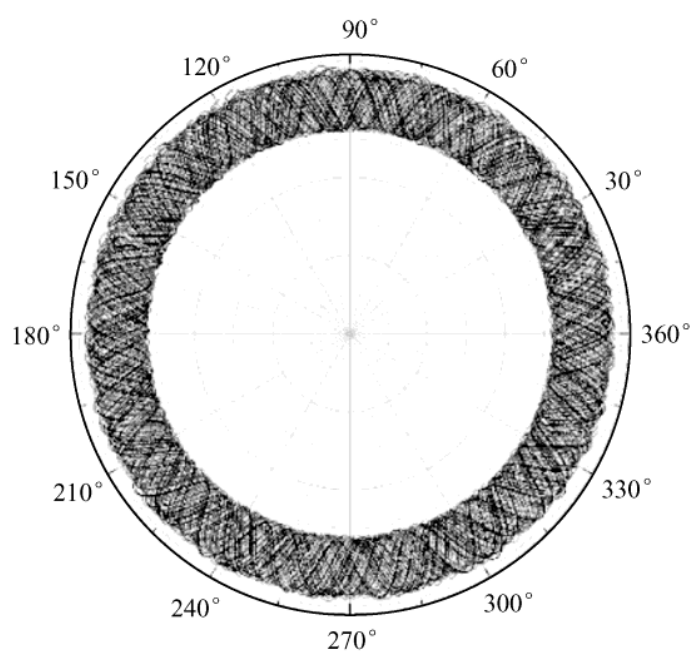

(b) 工况b

图 17 车轮多边形磨耗沿圆周相位分布

通过本章关于钢轨波磨激励下车轮多边形磨耗 发展的关键条件分析可知, 需要同时满足以下三个
条件, 多边形磨耗才能发展起来: (1) 钢轨波磨的波 长恰好整分车轮周长; (2) 线路钢轨波磨的波长尽可 
能为一个固定值, 或者在极小的范围内出现偏差;

(3) 车辆每次通过钢轨波磨区段时, 车轮均以同一位 置开始和钢轨波磨进行接触, 即车轮每次重复滚过 时接触位置相同。

很显然, 在实际条件下, 这三个条件均难以满 足。因此, 虽然钢轨波磨能够使轮轨力和蠕滑力沿 圆周呈周期性波动, 但是不是导致车轮多边形磨耗 产生的根本原因。

另外, 文献[19-20]研究了考虑转向架系统的柔 性共振后轮轨力和蠕滑率情况。可以看出, 当考虑 转向架系统的柔性变形后, 即使车辆运行于无波磨 的钢轨上, 轮轨力和蠕滑率也会出现周期性波动, 是否会产生多边形磨耗还有取决于列车运行速度、 车轮直径和激励频率三者是否满足多边形磨耗产生 的条件 ${ }^{[13]}$ 。

\section{5 结论}

本文以我国某线路上运行的高速动车组列车为 研究对象, 通过建立车轮多边形磨耗仿真预测模型, 结合现场试验数据, 调查钢轨波磨对车轮多边形磨 耗产生和发展的影响。

钢轨波磨虽然可以导致轮轨法向力和蠕滑率等 磨耗关键参数沿车轮圆周发生周期性波动。但是在 车辆实际运营条件下，钢轨波磨导致车轮多边形磨 耗产生这一观点的成立条件极为 “苛刻”, 对车轮和 钢轨波磨区段起始点的接触位置、钢轨波磨的波长 以及车轮周长都有严格的要求, 在实际中该条件难 以出现, 因此, 钢轨波磨的存在并不是车轮多边形 产生的根本原因。

\section{参 考 文 献}

[1] JOHANSSON A. Out-of-round railway wheels-literature survey, field tests and numerical simulations[D]. Gotoborg: Chalmers University of Technology, 2003.

[2] NIELSEN J, JOHANSSON A. Out-of-round railway wheels-A literature survey[J]. Proceedings of the Institution of Mechanical Engineers, Part F: Journal of Rail and Rapid Transit, 2000， 214 (2): 79-91.

[3] 温泽峰. 钢轨波浪形磨损研究[D]. 成都: 西南交通大 学, 2006 .

WEN Zefeng. Study on rail corrugation[D]. Chengdu: Southwest Jiaotong University, 2006.

[4] KAPER H. Wheel corrugation on Netherlands railway (NS): origin and effects of 'polygonization' in particular[J].
Journal of Sound \& Vibration, 1988, 120(2): 267-274.

[5] MORYS B, KUNTZE H, HIRSCH U. Investigation of origin and enlargement of out-of-round phenomena in high speed ICE-wheelsets[C]// In Proceedings of 10th European ADAMS Users' Conference, Frankfurt, 1995: 14.

[6] MORYS B, KUNTZE H. Simulation analysis and active compensation of the out-of-round phenomena at wheels of high speed trains $[\mathrm{C}] / /$ Proceedings of World Congress on Railway Research, Florence, Italy, 1997: 95-105.

[7] MORYS B. Enlargement of out-of-round wheel profiles on high speed trains[J]. Journal of Sound and Vibration, 1999, 227(5): 965-978.

[8] MEINKE P, MEINKE S, SZOC T. On dynamics of rotating wheel/rail systems in a medium frequency range $[\mathrm{C}] / /$ 4th German-polish workshop on dynamical problems in mechanical systems, Warszawa, Poland, 1996.

[9] MEYWERK M. Polygonalizaiton railway wheels[J]. Archive of Applied Mechanics, 1999, 69 (2): 102-120.

[10] 陈光雄, 金学松, 邬平波, 等. 车轮多边形磨耗机理的 有限元研究 $[J]$. 铁道学报, 2011，33(1): 14-18.

CHEN Guangxiong, JIN Xuesong, WU Pingbo, et al. Finite element study on the generation mechanism of polygonal wear of railway wheels. Journal of the China Railway Society[J]. 2011, 33(1): 14-18.

[11] JIN Xuesong, WU Lei, FANG Jianying, et al. An investigation into the mechanism of the polygonal wear of metro train wheels and its effect on the dynamic behaviour of a wheel/rail system[J]. Vehicle System Dynamics, 2012, 50(12): 1817-1834.

[12] 李伟, 李言义, 张雄飞, 等. 地铁车辆车轮多边形的机 理分析[J]. 机械工程学报，2013，49(18)：17-22.

LI Wei, LI Yanyi, ZHANG Xiongfei, et al. Mechanism of the polygonal wear of metro train wheels[J]. Journal of Mechanical Engineering, 2013, 49(18): 17-22.

[13] WU Yue, DU Xing, ZHANG Heji, et al. Experimental analysis of the mechanism of high-order polygonal wear of wheels of a high-speed train[J]. Journal of Zhejiang University, 2017, 18(8): 579-592.

[14] 吴越, 韩健, 刘佳, 等. 高速列车车轮多边形磨耗对轮 轨力和转向架振动行为的影响 [J]. 机械工程学报, 2018 , 54(4): 37-46.

WU Yue, HAN Jian, LIU Jia, et al. Effect of high-speed train polygonal wheels on wheel/rail contact force and 
bogie vibration [J]. Journal of Mechanical Engineering, 2018, 54(4): 37-46.

[15] 翟婉明. 车辆一轨道耦合动力学 $[\mathrm{M}] .3$ 版. 北京: 科学 出版社, 2007.

ZHAI Wanming. Vehicle-track coupled dynamics [M]. 3rd ed. Beijing: Science Press, 2007.

[16] ZHONG Shuoqiao, XIONG Jiayang, XIAO Xinbiao, et al. Effect of the first two wheelset bending modes on wheel-rail contact behavior[J]. Journal of Zhejiang University-SCIENCE A，2014， 15(12): 984-1001.

[17] XIAO Xinbiao, LING Liang, JIN Xuesong. A study of the derailment mechanism of a high speed train due to an earthquake[J]. Vehicle System Dynamics, 2012, 50(3): 449-470.

[18] HAN J, ZHONG S, XIAO X, et al. High-speed wheel/rail contact determining method with rotating flexible wheelset and validation under wheel polygon excitation [J]. Vehicle System Dynamics, 2017, 1-17.

[19] ZHONG Shuoqiao, XIAO Xinbiao, WEN Zefeng, et al. Effect of the wheelset's flexibility on wheel-rail contact behavior and a specific coupling of wheel-rail contact to the flexible wheelset[J]. Acta Mechanica Sinica，2016，32(2): 1-13.

[20] 钟硕乔. 车辆轨道耦合系统中的旋转柔性轮对建模[D]. 成都: 西南交通大学, 2018.

ZHONG Shuoqiao. Study on modelling of rotating flexible wheelset in vehicle-track dynamic system[D]. Chengdu: Southwest Jiaotong University, 2018.

作者简介: 吴越, 男, 1992 年出生, 博士研究生。主要研究方向为高速 列车轮轨关系与振动噪声。

E-mail:249368594@qq.com 